\title{
Single-sided Time Domain-Nuclear Magnetic Resonance to Study the Effect of Cell Membrane Electroporation on the Water Mobility in Vegetal Tissues
}

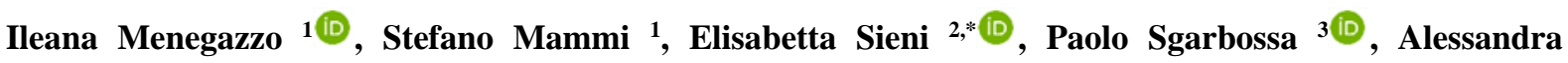

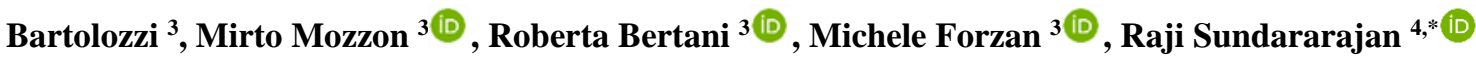 \\ Padova University, Department of Chemical Sciences via Marzolo 1, 35131 Padova, Italy \\ Insubria University, Department of Theoretical and Applied Sciences via Dunant, 321100 Varese, Italy \\ Padova University, Department of Industrial Engineering, via Marzolo, 9, 35131 Padova, Italy \\ School of Engineering Technology, Purdue University, Knoy Hall of Technology, 401 N. Grant St. West Lafayette, IN \\ 47907, USA \\ * Correspondence: elisabetta.sieni@ uninsubria.it (E.S.), raji@ purdue.edu (R.S.);
}

Scopus Author ID 7004860497

Received: 15.01.2021; Revised: 28.02.2021; Accepted: 2.03.2021; Published: 7.03.2021

\begin{abstract}
This paper presents the characteristics of potato and apple tissues, with/out electroporation, by Time Domain-Nuclear Magnetic Resonance (TD-NMR). A portable TD-NMR was used to measure the proton relaxation time, $\mathrm{T} 2$, and the changes in the cells due to molecular water mobility of potato tubers,-NMR to identify the modifications that occurred at the cell level involving water molecules mobility in potato tubers and apple tissues after the electroporation treatment and compared with nonelectroporated ones. The comparisons with normal potato and apple tissue and preliminary measurements in bulk were performed. Samples were also analyzed in terms of conductivity of the tissue and microscopic morphology. The results indicate that the electroporation process effect is identified with a variation of the peak position in T2 distribution, associated with sub-cell modifications.
\end{abstract}

Keywords: water mobility; electroporation; vegetal tissue; potato; time domain Nuclear-Magnetic Resonance (NMR); cell membrane.

(C) 2021 by the authors. This article is an open-access article distributed under the terms and conditions of the Creative Commons Attribution (CC BY) license (https://creativecommons.org/licenses/by/4.0/).

\section{Introduction}

Electroporation involves the targeted application of high magnitude, short width electrical pulses (EP) across the cell memabrane, which causes accumulation of charges and hence enhnaces membrane voltage, $V m$. When $\mathrm{Vm}$ is around 0.5 to $1 \mathrm{~V}$, across its $5 \mathrm{~nm}$ thickness, there is a several-fold enhancement of electric field $(100 \mathrm{MV} / \mathrm{cm})$, leading to the pore formation [1-10].

During electroporation, drug molecules can enter the cancer cells in various ways, such as diffusion, electro-osmotic, and colloid-osmotic flow [11]. The flow of light and small drug molecules due to electroporation is given by Fick's equation [12]:

$$
F_{S}(t)=P_{S} x(N, T) A / 2\left(1-\frac{E p}{E}\right) \Delta S e^{-k(N, T)^{i}}(1)
$$

where Fs is the flow of molecules $\mathrm{S}$ diffusing through the plasma membrane, Ps is the permeation coefficient of the molecule $S$ across the membrane, $x$ is a function, which represents the probability of $(0<x<1)$, A is cell surface, $\mathrm{E}$ is the applied electric field intensity, Ep is the 
threshold for permeabilization, $\Delta \mathrm{S}$ is concentration difference of $\mathrm{S}$ between the cell and external medium, $\mathrm{k}$ is the time constant of the resealing process, and $\mathrm{t}$ is the time after the pulse.

The pore formation is a two-step process [13]. First, small and non-conducting hydrophobic pores form in the cell membrane. Second, these hydrophobic pores expand in size, and lipid molecules invert on the edges to create conducting hydrophilic pores. The energy of pore formation $(\mathrm{E}(\mathrm{r}))$ for both types of pores is a function of the pore radius (r) and $V_{m}$, as shown in (1) for hydrophobic and (2) for hydrophilic pores, respectively [13].

$$
\begin{aligned}
& \left.E(r)=2 \pi h r \sigma o(\infty)\left[I_{1}\left(r / r_{0}\right) / I_{0}\left(r / r_{0}\right)\right]\right)-\pi a_{p} V_{m}^{2} r^{2} \\
& E(r)=2 \pi \gamma r-\int_{0}^{r} 2 \pi \Gamma\left(r^{*}\right) r^{*} d r^{*}+(C / r)^{4}-\pi a_{p} V_{m}^{2} r^{2}
\end{aligned}
$$

where $\mathrm{h}$ is the membrane thickness, $\sigma_{0}(\infty)$ is a constant $\left(5 \cdot 10^{-2} \mathrm{~N} / \mathrm{m}\right), \mathrm{r} 0$ is the characteristic length, $\mathrm{I}_{0}$ and $\mathrm{I}_{1}$ are $0^{\text {th }}$ and $1^{\text {st }}$ order modified Bessel functions, $\gamma$ is the strain energy per unit length of the bilayer pore edge or perimeter, $\Gamma$ is the surface energy per unit area of the intact bilayer, $\mathrm{C}$ is a constant $\left(9.67 \cdot 10^{-15} \mathrm{~J}^{1 / 4} \mathrm{~m}\right)$, ap is the property of the membrane and its aqueous environment, expressed in terms of $h$ and permittivity of water $\left(\varepsilon_{\mathrm{w}}\right)$ and membrane $\left(\varepsilon_{\mathrm{m}}\right)$, as ap $=\left(\varepsilon_{\mathrm{W}}-\varepsilon_{\mathrm{m}}\right) /[2 \mathrm{~h}]$.

This two-step pore formation phenomenon is called electroporation, and it can enhance the uptake of external molecules up to 1000 times [14]. When electroporation is applied towards chemotherapeutics' uptake, this non-surgical procedure, known as Electrochemotherapy (ECT), is gaining momentum as an alternative to conventional therapies for advanced, inoperable, and radio- and chemo-resistant tumors of all histological types of cutaneous and subcutaneous metastases in the EU [15]. This phenomenon is evidenced by an increment of the tissue's conductivity [1, 16-18]. This technique is applied in medicine to improve the chemotherapeutic drug uptake and the food industry to treat fruits to enhance juice extraction or in low-temperature sterilization [19-25]. This technique is also used to study the effect of electrical pulses on vegetal tissues. Towards this, numerous studies have shown that Time Domain-Nuclear Magnetic Resonance (TD-NMR) is an excellent method to characterize water mobility and water distribution in food items, such as meat, fish, cheese, cereals, fruits, and vegetables, including potatoes [26-39].

The basic physical rationale behind the generation of the NMR signal is the same in all kinds of NMR instruments, and it derives from the NMR-active atomic nuclei present in the sample. Time Domain NMR applications include the measurements of the relaxation properties of protons that, after excitation by a radiofrequency pulse, return to the equilibrium condition: energy is lost to the surroundings in the form of heat (spin-lattice relaxation), and coherence among spins is also lost (spin-spin relaxation).

The 1H NMR spin-lattice $\left(\mathrm{T}_{1}\right)$ and spin-spin $\left(\mathrm{T}_{2}\right)$ relaxation times of water in vegetable tissue have been used to describe the water status. Whereas bulk water exhibits a single value for each relaxation time, water compartmentalized in subcellular structures, such as vegetable tissues, exhibits multiple relaxation time values. Water molecules or protons are in exchange among these compartments, and the exchange rates are controlled by the permeability of the membranes separating the compartments, their size, and/or by the diffusion processes of water molecules.

Whereas differences in the longitudinal relaxation times $\left(\mathrm{T}_{1}\right)$ are usually not sufficient to identify water in different compartments, differences in transverse relaxation times $\left(\mathrm{T}_{2}\right)$ are more pronounced. Moreover, the total proton NMR signal from any sample region is directly proportional to the proton density in that region, providing quantitative information about water compartmentation. 
The literature reports that in vegetable cells, the mean distribution of $\mathrm{T}_{2}$ relaxation times includes three populations, partially overlapped, associated with three proton compartments. Specifically, the water populations could be assigned to water located in the cell wall, cytoplasmic water, and water in the vacuole (with the longest $\mathrm{T}_{2}$ relaxation value) $[40,41]$. In potato cells, another proton population with the lowest $\mathrm{T} 2$ relaxation value is evident, and it can be assigned to water on the surface or inside starch granules [42].

Changes in water NMR dynamic properties during ripening, processing, and storage operations on fruits and vegetables can, in principle, reveal sub-cellular modifications and contribute to a microscopic understanding of these processes [43]. Examples of the application of this approach are present in the literature [44-47].

A special kind of TD-NMR was applied in the present work, that is, the Mobile Universal Surface Explorer (Single-sided TD-NMR) [48, 49]. This portable, single-sided NMR device has been designed to evaluate the proton content of objects non-invasively and nondestructively. Specifically, we used it to measure the proton relaxation time and study the associated mobility of water molecules before and after the electroporation process on potato tissue samples of different varieties. Comparisons with apple tissue and preliminary measurements in a bulk TD-NMR instrument (Minispec) were also performed.

The T2 transverse relaxation times measured with the Single-sided TD-NMR were shown to be sensitive to the electroporation process. Preliminary studies with the NMRMinispec and microscope observations confirmed the data and suggested a deeper comprehension of the process at the subcellular level.

\section{Materials and Methods}

Four different types of potato tubers, P1, P2, P3, and P4, and one type of apple were purchased from the local market. These different types of potato tubers belong to different varieties with clear or red peel and yellow core among those available at the experiments' time. The apple variety was the one available at the time of the experiment. The experiments were conducted in July. Each vegetable sample, either electroporated or non-electroporated, was analyzed using the Single-sided TD-NMR. Each side of the treated sample was analyzed to verify if the electroporation produced differences in terms of the water molecule's mobility on the surface. The same analysis was applied to the non-electroporated samples to determine the differences by comparison.

For each sample type, the relaxation time $\mathrm{T} 2$ was also measured in the bulk instrument Minispec to better understand the Single-sided TD-NMR results.

Electroporated tissues were prepared for optical microscope visualization. Treated samples were observed one hour after electroporation. For each piece, both sides, top, and bottom were sliced and observed under a microscope to evaluate the cell membrane morphology. Non-treated pieces were also analyzed with the microscope for comparison.

$24 \mathrm{~h}$ after treatment, the treated samples were observed for any color change and other electroporation effects.

\subsection{Electroporation.}

From each of the potato and apple, six parallelepiped pieces with a size close to $5 \mathrm{x} 10$ $\mathrm{x} 30 \mathrm{~mm}$ were cut. Three of the pieces were treated with the electroporation voltage pulses, and the other three were untreated controls. The voltage pulses were applied using the EPSO2 
voltage pulse generator (IGEA SpA, Carpi, MO, Italy) connected to a pair of parallel stainless steel plate electrodes with a gap of $5 \mathrm{~mm}$, positioned, as shown in the set-up, in Fig. 1 (a) [50, $51]$.

To each sample, a sequence of 24 unidirectional voltage pulses, with a suitable amplitude in the order of $1000 \mathrm{~V} / \mathrm{cm}(500 \mathrm{~V})$ for potato and in the order of $2000 \mathrm{~V} / \mathrm{cm}(1000$ V) for apple tissue were applied to one of the $10 \times 30 \mathrm{~mm}$ sides. For each sample, the area facing the positive electrode was marked. A and the one facing the negative electrode were marked B.

The two sides, the treated and the untreated one, were then analyzed using the SINGLESIDED TD-NMR probe. Finally, the sample's two sides were sliced, dyed with Methylene Blue, and observed at the optical microscope. The data of the electroporated samples were compared with those of the non-electroporated tissues.

\subsection{Water content.}

For each sample, the water content was measured by drying the samples in a static oven (Mod. 2100 High-Performance Oven, F.lli Galli) according to the following procedure: (i) three samples of the same vegetable was cut; (ii) each sample was weighed (HR-200 Scientific Balance, A\&D Instruments), obtaining the value, $\mathrm{m}_{\text {fresh; }}$ (iii) the samples were dried for $24 \mathrm{~h}$ in a static oven at $100{ }^{\circ} \mathrm{C}$; (iv) the same samples were weighted till constant weight, obtaining the dry weight, $\mathrm{m}_{\mathrm{dry}}$; (v) finally, the water content was computed as a percent using equation (1):

$$
\text { watercontent }[\%]=100 \frac{m_{\text {fresh }}-m_{\text {dry }}}{m_{\text {fresh }}}
$$

\subsection{Tissue morphology.}

The tissue morphology before and after electroporation was studied using an inverted microscope (Olympus IX50). A thin layer from each side of the potato and apple parallelepiped was cut in the electroporated area using a cutter. The larger section of the parallelepiped was chosen. The slices were washed using deionized water and immersed in $0.2 \%$ Methylene blue in deionized water (115943 Methylene blue C.I. 52015, Merck Chemicals) for $1 \mathrm{~min}$. The pieces were washed again in deionized water and then placed on a microscope glass slide covered with a coverslip. Every slice was washed again by capillarity to remove the excess dye and was observed using an Olympus IX50 microscope with a magnification of 400x and a Canon EOS 1100D camera focusing on the cell membrane.

\subsection{Single-sided TD-NMR vegetable tissue conductivity.}

The vegetable tissue conductivity could be described as a non-linear function of the electric field intensity, $\sigma(\mathrm{E})$, as in $[50,52]$ :

$$
\sigma(\mathrm{E})=\sigma_{0}+\frac{\sigma_{1}-\sigma_{0}}{2}\left(1+\tanh \left(\mathrm{k}_{\mathrm{v}}\left(\mathrm{E}-\mathrm{E}_{\mathrm{th}}\right)\right)\right)
$$

where $\sigma_{0}$ and $\sigma_{1}[\mathrm{~S} / \mathrm{m}]$ is the conductivity at $0 \mathrm{~V} / \mathrm{cm}$, and at the maximum value of $\mathrm{E}$ (e.g., $1000 \mathrm{~V} / \mathrm{cm})$, respectively, $\mathrm{k}_{\mathrm{v}}[\mathrm{m} / \mathrm{V}]$ is a constant and $\mathrm{E}_{\mathrm{th}}[\mathrm{kV} / \mathrm{m}]$ is the electric field.

The electrical conductivity, $\sigma$, of the vegetable tissues was evaluated experimentally, using a suitable multi-box device, with 8 wells. Each well has a rectangular section $(11 \times 9 \mathrm{~mm})$ and is $11.3 \mathrm{~mm}$ thick, as described in (Fig. 1 (b)). The voltage pulses were applied using a plate electrode formed by two stainless steel plates with rectangular geometry with the side $10 \mathrm{~mm}$ 
long and distant $\mathrm{L}=7 \mathrm{~mm}$ (covering all the box depth of $11.3 \mathrm{~mm}$ ). The two plates were inserted into each box.

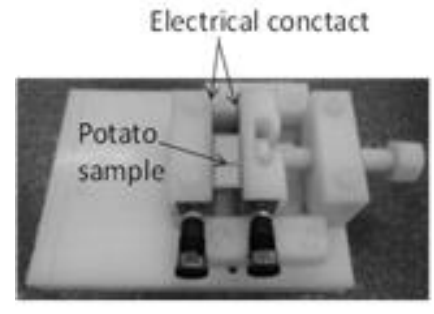

(a)

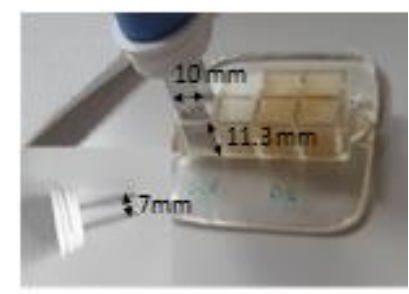

(b)

Figure 1. Experimental set-ups used for (a) the treatment with voltage pulses of the samples for the TD-NMR experiment and (b) for the evaluation of the electrical conductivity of the vegetable tissue [53].

Eight voltage pulses were applied to the electrode plates using the voltage pulse generator. The amplitude of the voltage pulses was varied from $100 \mathrm{~V}(\mathrm{E}=143 \mathrm{~V} / \mathrm{cm})$ to 1000 $\mathrm{V}(\mathrm{E}=1370 \mathrm{~V} / \mathrm{cm})$. Voltage and current values were recorded and analyzed to compute the resistance at the electrodes and the material's corresponding conductivity as in [30]. The conductivity was computed using Ohm's law in equation (3), and the model for the conductivity, $\sigma$, of a parallelepiped with section $\mathrm{A}=10 \times 11.3 \mathrm{~mm}$ and length $\mathrm{L}=7 \mathrm{~mm}$ :

$$
\sigma=\frac{1}{R} \frac{L}{A}
$$

The measured conductivity values as a function of the electric field intensity were fitted using the non-linear least mean square method to find the parameters of equation (2) [50, 54].

\subsection{TD-NMR.}

$\mathrm{T}_{2}$ measurements were carried out using a Bruker compact spectrometer (Minispecmqseries). When equipped with a Profiler with a $0 \mathrm{~mm}$ depth probe, the single-sided TD-NMR surface has a Larmor frequency of $15.50 \mathrm{MHz}$. The samples were analyzed at room temperature using a multi-echo CPMG-type pulse sequence with echo times of $100 \mu \mathrm{s} ; 2500$ echoes were recorded $[55,56]$. For bulk $\mathrm{T}_{2}$ measurements, the Bruker Minispec magnet was equipped with a $10 \mathrm{~mm}$ temperature-controlled probe with $6 \mu \mathrm{s}$ dead time. The magnetic field strength was $0.47 \mathrm{~T}$, corresponding to a resonance frequency for protons of $19.95 \mathrm{MHz}$. The samples were analyzed at $22{ }^{\circ} \mathrm{C}$, using the Carr-Purcell-Meiboom-Gill (CPMG) sequence with a $\tau$ value of $150 \mu$ s. Data from 10000 echoes were acquired for 16 or 32 scans for each measurement.

The relaxation times of the different components were extracted from multiexponential decay curves using the program Uniform PENalty (UPEN), based on the inverse Laplace transform developed by Fantazzini and co-workers (Upenwin ver. 1.04) [57, 58].

UPEN inverts the CPMG signal searching for the least biased distribution of relaxation times according to the following equation: $I(2 \tau n)=\Sigma i I_{0}\left(T_{2, i}\right) \exp \left(-2 \tau n / T_{2, i}\right)$, where $2 \tau$ is the CPMG interpulse spacing, $\mathrm{n}$ is the index of a CPMG echo, and $\mathrm{I}_{0}\left(\mathrm{~T}_{2, \mathrm{i}}\right)$ is the signal intensity of the $T_{2, i}$ component at $\tau=0$, sampled logarithmically in the interval between $T_{2, \min }$ and

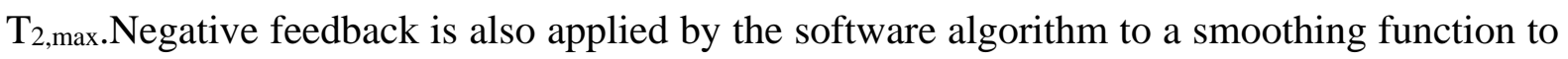
prevent excessive details in the distribution of relaxation times. As a consequence, the resolution of features is lowered as a default [59]. The major advantage of this approach concerning other fitting models (such as the Levenberg-Marquardt one) is that an a priori knowledge about the number of $T_{2} \mathrm{~S}$ is unnecessary.

In the present work, the default UPEN parameters were generally adopted. 


\subsection{Statistical analysis.}

Statistical analysis on measurements data was performed using an ANOVA test to compare the values of the relevant peaks of the $\mathrm{T}_{2}$ time measured in different conditions.

\section{Results and Discussion}

Fig. 2 (a) and (b) show the non-electroporated tissue of a potato tuber. Here, arrow ' 1 ' points to the cell wall of a typical potato cell with polygonal shape, arrow ' 2 ' to the starch granules, and arrow ' 3 ' to an area where the cells are smaller and packed, and the white star represents the vacuole structure inside the cell. Differences in the tissue morphology (dimensions of cells and vacuoles or different starch granules packing) could cause differences in recorded values. It is well known that the potato tubers also show inhomogeneity in tissue morphology. Comparatively, the apple tissue is more homogeneous concerning potato tissue, and no starch granules are present, as shown in Fig. 2 (c).

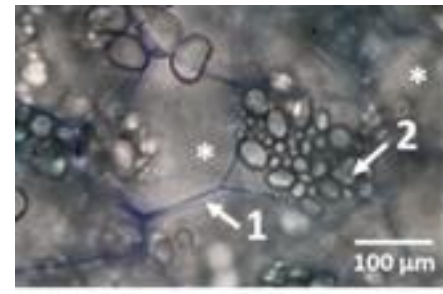

(a)

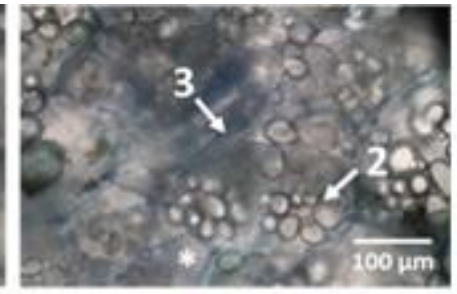

(b)

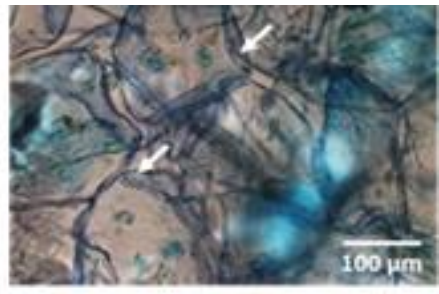

(c)

Figure 2. (a) and (b) Potato morphology for sample P1 (left) and P2 (right) and (c) apple tissue morphology. Stars identify the vacuole structure, where, arrow ' 1 ' shows the cell wall, arrow ' 2 ' shows the starch granules, and arrow 3 shows the area where the cells are smaller and packed.

To determine the electric field needed to electroporate the analyzed tissue's cell membrane, the sample conductivity was evaluated as in [24]. The electric field intensity was chosen to maximize conductivity and guarantee the tissue's electroporation for any higher field intensity.

The parameters of the curve of the vegetable tissue conductivity described in equation (2) as a function of the electric field intensity are obtained by fitting the measurement data obtained applying incremental voltage amplitude to potato or apple cube as described in Materials and Methods. The measured conductivities for each applied voltage for the four potato types and one apple are reported in Fig. 3 (a) and (b), respectively. Data obtained from the different potato samples are identified with different symbols (P1, crosses; P2, triangles; P3, circles; and P4, dots). Fig. 3(c) shows two examples of the treated potato samples $24 \mathrm{~h}$ after the treatment and the color changes due to electroporation, whereas Fig. 3(d) shows the apple samples treated with incremental electric field intensity observed $24 \mathrm{~h}$ after electroporation. The four different types of potatoes show differences in terms of conductivity. At an electric field of $1000 \mathrm{~V} / \mathrm{cm}$, all the potato tubers show their maximum conductivity values, indicating that the field intensity chosen to treat the potato slices analyzed with Single-sided TD-NMR is suitable to electroporate the tissues. In the case of the apple samples, the maximum conductivity is reached using an electric field intensity of $970 \mathrm{~V} / \mathrm{m}$; therefore, at $2000 \mathrm{~V} / \mathrm{cm}$, the electric field intensity used for the treatment of the apple slices analyzed with the Singlesided TD-NMR, the tissue could be considered electroporated. 
The conductivity values measured in the experimental set-up in Fig. 1(b) and evaluated at each electric field amplitude are reported in Table 1, whereas Table $2 \mathrm{~A}$ reports the values of the four parameters of the model (2) evaluated for each potato tuber or apple sample. Table $2 \mathrm{~B}$ also reports the water content evaluated for each sample used in the experiments as determined by equation (1). The potatoes' water content was close to $80 \%$ in three of the samples, whereas the last potato tuber showed a higher value of $83 \%$. The ANOVA analysis would have a pvalue of 0.086 for all the potato tubers compared and a p-value of 0.27 if the P1, P2, and P3 samples were considered, indicating that the values are statistically not significantly different $(\mathrm{p}=0.05)$. Quantitatively, the samples $\mathrm{P} 1, \mathrm{P} 2$, and $\mathrm{P} 3$ have a comparable water content, whereas the water content of the sample P4 is higher. In apple samples, the water content was $83 \%$.

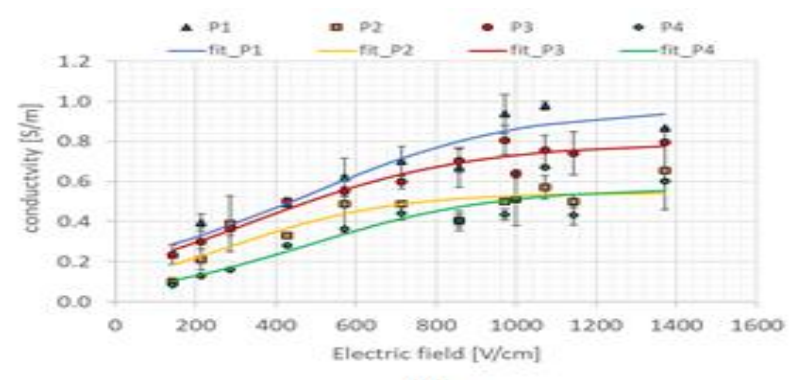

(a)

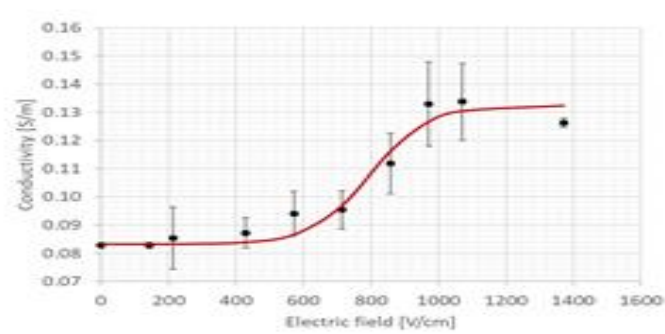

(b)

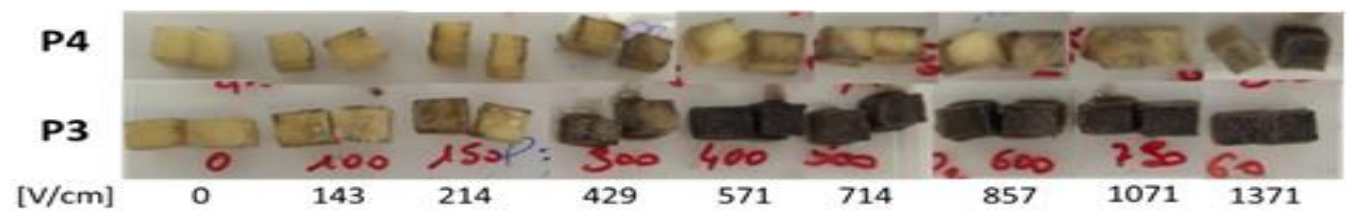

(c)

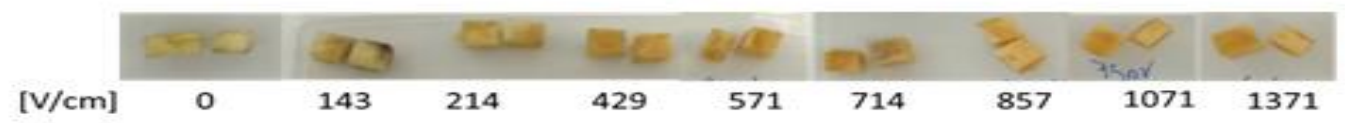

(d)

Figure 4. Conductivity in $[\mathrm{S} / \mathrm{m}]$ as a function of the electric field amplitude in $[\mathrm{V} / \mathrm{cm}]$ for (a) the four tubers analyzed and (b) apple tissue. (b) Pictures of the vegetable pieces electroporated at different electric field intensities taken $24 \mathrm{~h}$ after treatment: (c) two representative samples, P2 and P3, of potato tubers and (d) apple tissue.

Table 1. Conductivity in $[\mathrm{S} / \mathrm{m}]$ measured at different electric field amplitudes in $[\mathrm{V} / \mathrm{cm}]$ with standard deviation

\begin{tabular}{|c|c|c|c|c|c|c|c|c|c|c|}
\hline $\mathrm{E}[\mathrm{V} / \mathrm{cm}]$ & $\begin{array}{c}\mathbf{P 1} \\
\sigma[\mathbf{S} / \mathbf{m}]\end{array}$ & std $(\sigma)$ & $\begin{array}{c}\mathbf{P 2} \\
\sigma[\mathbf{S} / \mathbf{m}]\end{array}$ & std $(\sigma)$ & $\begin{array}{c}\mathbf{P 3} \\
\sigma[\mathbf{S} / \mathbf{m}]-\end{array}$ & $\operatorname{std}(\sigma)$ & $\begin{array}{c}\mathbf{P 4} \\
\sigma[\mathrm{S} / \mathbf{m}]\end{array}$ & $\operatorname{std}(\sigma)$ & $\begin{array}{c}\mathbf{A 1} \\
\sigma[\mathrm{S} / \mathbf{m}]\end{array}$ & $\operatorname{std}(\sigma)$ \\
\hline 143 & 0.23 & \pm 0.050 & 0.10 & \pm 0.017 & 0.23 & \pm 0.050 & 0.08 & \pm 0.000 & 0.083 & \pm 0.000 \\
\hline 214 & 0.40 & \pm 0.043 & 0.21 & \pm 0.052 & 0.30 & \pm 0.074 & 0.13 & \pm 0.010 & 0.083 & \pm 0.000 \\
\hline 429 & 0.49 & \pm 0.001 & 0.33 & \pm 0.012 & 0.50 & \pm 0.015 & 0.28 & \pm 0.011 & 0.087 & \pm 0.005 \\
\hline 571 & 0.62 & \pm 0.096 & 0.49 & \pm 0.143 & 0.55 & \pm 0.024 & 0.36 & \pm 0.000 & 0.094 & \pm 0.008 \\
\hline 714 & 0.70 & \pm 0.075 & 0.49 & \pm 0.000 & 0.60 & \pm 0.034 & 0.44 & \pm 0.033 & 0.095 & \pm 0.007 \\
\hline 857 & 0.67 & \pm 0.098 & 0.41 & \pm 0.029 & 0.70 & \pm 0.059 & 0.40 & \pm 0.049 & 0.112 & \pm 0.011 \\
\hline 971 & 0.94 & \pm 0.097 & 0.50 & \pm 0.005 & 0.81 & \pm 0.073 & 0.44 & \pm 0.028 & 0.133 & \pm 0.015 \\
\hline 1071 & 0.98 & \pm 0.022 & 0.57 & \pm 0.059 & 0.76 & \pm 0.073 & 0.67 & \pm 0.004 & 0.134 & \pm 0.01 \\
\hline 1371 & 0.87 & \pm 0.000 & 0.65 & \pm 0.194 & 0.80 & \pm 0.007 & 0.60 & \pm 0.010 & 0.126 & \pm 0.00 \\
\hline
\end{tabular}

Table 2a. Values of the parameters of the conductivity model (2) for potato and apple samples.

\begin{tabular}{c|c|c|c|c} 
Sample & $\boldsymbol{\sigma}_{\mathbf{0}}[\mathbf{S} / \mathbf{m}]$ & $\boldsymbol{\sigma}_{\mathbf{1}}[\mathbf{S} / \mathbf{m}]$ & $\mathbf{k}_{\mathbf{v}}[\mathbf{c m} / \mathbf{V}]$ & $\mathbf{E}_{\text {th }}[\mathbf{k V} / \mathbf{c m}]$ \\
\hline P1 & 0.104 & 0.96 & 0.0012 & 480.7 \\
\hline P2 & 0.0001 & 0.54 & 0.0024 & 280.9 \\
\hline P3 & 0.0001 & 0.79 & 0.0019 & 340.1 \\
\hline P4 & 0.0001 & 0.56 & 0.0021 & 478.2 \\
\hline A1 & 0.083 & 0.132 & 0.0058 & 797.1
\end{tabular}


Table 2b. Water content [\%] for potato and apple samples.

\begin{tabular}{c|c|c} 
Sample & Water content [\%] & Std [\%] \\
\hline P1 & 79.4 & \pm 0.3 \\
\hline P2 & 79.5 & \pm 1.1 \\
\hline P3 & 80.6 & \pm 0.4 \\
\hline P4 & 83.0 & \pm 0.7 \\
\hline A1 & $83 \%$ & \pm 0.7
\end{tabular}

The potato and apple tissues treated with the electric field observed $24 \mathrm{~h}$ after electroporation appear dark compared to the non-treated ones, which maintained their original yellow color. Fig. 4 shows the potato and apple pieces $24 \mathrm{~h}$ after pulse application. In potato samples, the color after electroporation is between dark brown and black. Specifically, the color change after electroporation of samples $\mathrm{P} 1$ and $\mathrm{P} 2$ is less intense than the one that occurred in samples P3 and P4. Electroporation of the apple sample caused the color to become clear brown from the original yellow of the non-electroporated sample.

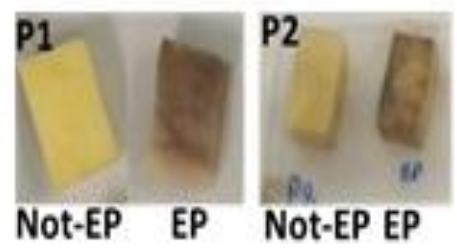

(a)

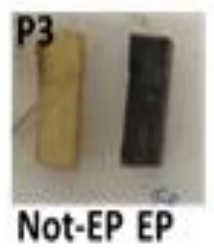

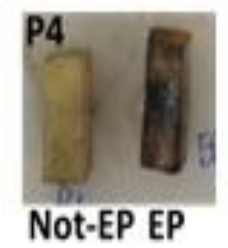

Not-EP EP

\section{Not-EP EP}

(b)

Figure 4. Vegetable pieces treated (labeled with EP) and non-treated were observed $24 \mathrm{~h}$ after the voltage pulses application. (a) potato, 4 tubers, and (b) apple.
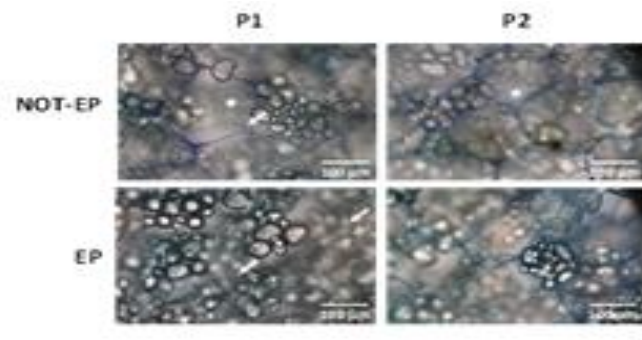

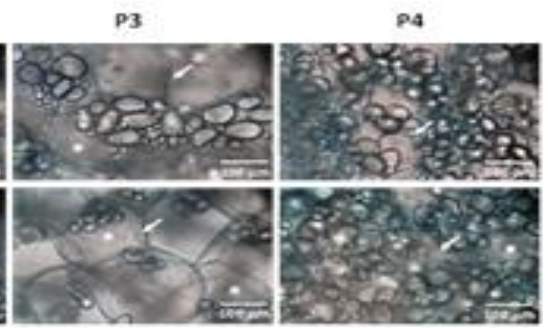

(a)

NOT-EP

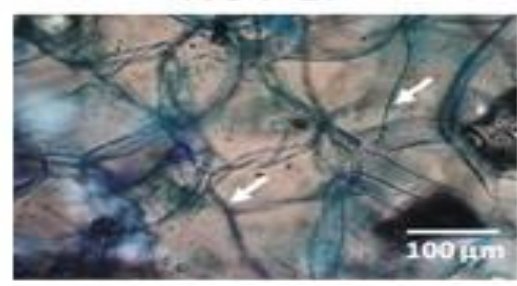

EP

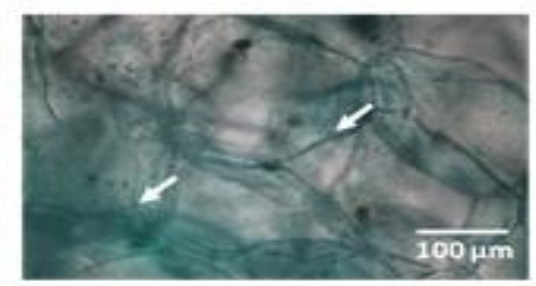

(b)

Figure 5. Microscope images of non-treated and electroporated (a) potato (4 tubers) and (b) for apple tissue. Arrows highlight membrane and cell wall, while stars the vacuole structure.

At the microscopic level, the images of the potato tissue (Fig.5 (a)) reveal well-defined cell membranes and cell walls, with a regular and square shape in the non-treated tissues, whereas they appear damaged and with an irregular shape in the electroporated tissue[60-65]. Here, the arrow in the electroporated samples (EP) shows the points where the cell membrane appears detached from the cell wall. In the non-electroporated samples' images, the cell membrane appears attached to the cell wall and shows a regular shape. The stars in both series 
of images show vacuole structures that are retracted in the electroporated cells. This could be related to the loss of liquid fraction. The tissue after electroporation shows a larger loss of liquid less than the one related to non-treated tissue observed at the same time. Fig. 5 (b) shows a comparison of the apple tissues before and after electroporation. In this case, the cell wall, marked with white arrows, is modified; cells appear to be of irregular shape, and the cell membrane detached from the cell wall.

Fig. 6 shows the Single-Sided TD-NMR T 2 values follow the typical distribution. The peak component at $150-160 \mathrm{~ms}$ and a broader one in the range $0.5-20 \mathrm{~ms}$ could be identified in all the samples, both treated and non-treated. The arrow with the star marks the 150-160 ms component. Comparing with the $\mathrm{T}_{2}$ profiles reported in the literature, we can assign the main peak to the water in the vacuole marked by the arrow on the right side (water in the vacuole shows the longest $\mathrm{T}_{2}$ value), and the other water populations are overlapped. The left two arrows indicate the contribution of water, not in vacuole towards T2. Specifically, the water populations could be assigned to water located on the cell wall, cytoplasmatic water, and water linked to starch granules [41-43].

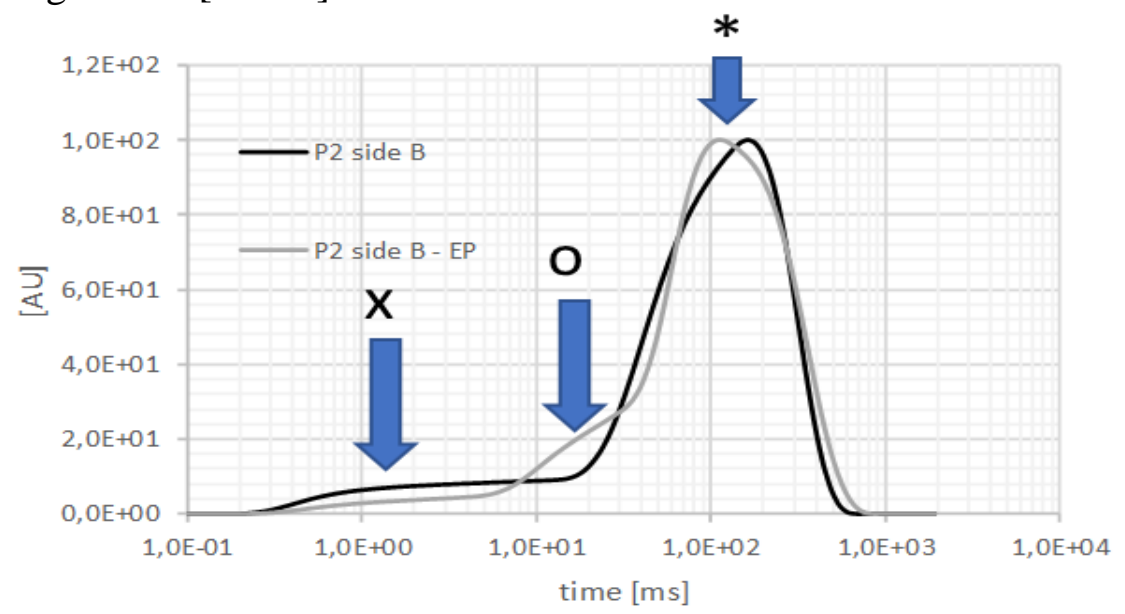

Figure 6. Typical inverse Laplace transform of the T2 signal for not-treated and treated potatoes, where the main peak is marked by the star and the other water components are marked by a cross and a circle.

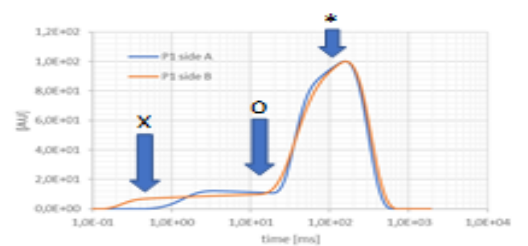

(a)

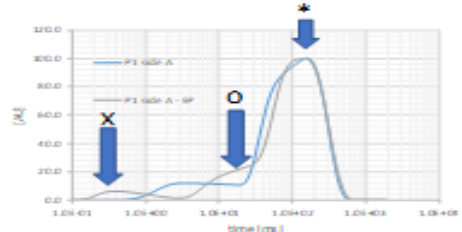

(b)

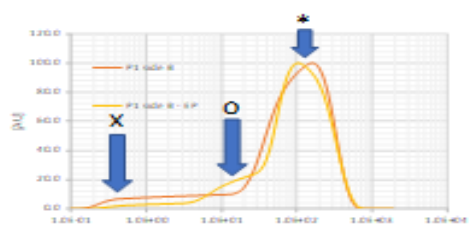

(c)

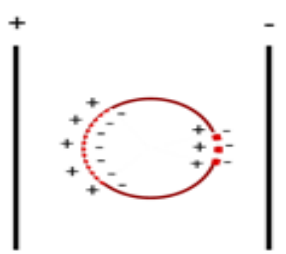

(d)

Figure 7. inverse Laplace transform of the T2 signal (a) for not-treated potato side A and B and for electroporated and non-electroporated potato (b) side A and (c) side B. Panel (d) represents the electroporation asymmetry if unipolar pulses are used.

In Fig. 6, the T2 relaxation value behavior at sides A and B of the sample is also reported. The $\mathrm{T}_{2}$ values show a difference in the area marked by the two arrows at the left. In fact, it is well known that the electroporation effect is different on the side facing the positive 
and the negative electrode if the pulses are applied in the same direction without reversing them periodically [11].

It must be noted that, due to the inhomogeneous magnetic field of the Single-sided TDNMR, the $T_{2}$ values measured in this work are shorter than the values previously reported for homogeneous TD-NMR, but some similar considerations apply.

In the case of electroporated potatoes, the first part of the profile changes, and the $\mathrm{T}_{2}$ relaxation value of the main population is lower.

Fig.7a-c shows the $\mathrm{T}_{2}$ distribution evaluated at side $\mathrm{A}$ and side $\mathrm{B}$ of electroporated and non-electroporated potato slices. In the non-treated potato, side A and B show a similar profile, whereas side A and B display differences in T2 distribution for the electroporated potato. The observed asymmetry could be related to the opposite polarity of the electrodes on the two sides as schematically represented in Fig 7 (d), where the side facing the positive electrode shows a larger electroporated area on the membrane surface with smaller pores, whereas on the side facing the negative electrode the electroporation area is smaller, but with larger pores [11]. Finally, considering electroporated samples, the area's peak marked a circle appears modified concerning both the signal related to the non-electroporated sample (side A and side B).

In Table 3, the values of the main $\mathrm{T}_{2}$ component for each side of the electroporated and non-electroporated potato pieces are reported. Both sides of non-electroporated potatoes show the same $T_{2}$ values. The electroporated pieces' peak maxima are shifted toward lower values, and the two sides of the electroporated potato show different peak shifts. The $\mathrm{T}_{2}$ of the positive side (A) is longer than the negative one (B). This result reflects the asymmetry of the electroporation process. The electroporation of cell membranes depends on the voltage polarity of the applied pulses. The side of the membrane facing a positive polarity shows a larger area of electroporation, whereas the side of the cell membrane facing the zero voltage electrode (negative polarity) experiments more intense electroporation in a smaller area, as shown in Fig. 7(b)-(c) [66]. Because the Single-sided TD-NMR detects water in the first layer (up to a maximum depth of $1 \mathrm{~mm}$ ), it can distinguish the two sides.

Table 3. Position of the main peak $[\mathrm{ms}]$ in electroporated and non-electroporated potatoes.

\begin{tabular}{c|c|c|c|c} 
& Side A & Side B & EP Side A & EP Side B \\
\hline P1 & 156 & 159 & 143 & 105 \\
\hline P2 & 162 & 162 & 166 & 113 \\
\hline P3 & 150 & 180 & 138 & 104 \\
\hline P4 & 156 & 151 & 139 & 134
\end{tabular}

Focusing on the values of the $\mathrm{T}_{2}$ main peak for the non-electroporated sides (columns 1 and 2) in Table 3 and using ANOVA, it appears that the two groups are homogenous (the pvalue is 0.32 , null hypothesis: $\mathrm{T}_{2}$ values for side $\mathrm{A}$ and side $\mathrm{B}$ are similar). Considering the electroporated side A of the potato piece and the corresponding non-electroporated one, the ANOVA analysis shows that the two groups are different (except for sample P2) and the pvalue is $<0.05$; nevertheless, the largest difference is observed on side B comparing the electroporated and the non-electroporated samples. In this case, the ANOVA analysis for the two groups of values shows a p-value $<0.05$ (0.001). Sides A and B analyzed after electroporation show a difference in $T_{2}$ value ( $p$-value $<0.05-$ null hypothesis: $T_{2}$ values for side A and side B are similar). This result enforces the hypothesis that the electroporation in unipolar pulses is different if the slice surface is faced with the positive or negative electrode.

The potato sample $\mathrm{P} 2$ was also analyzed in the more homogeneous magnetic field of the Minispec instrument (Fig. 8a). The measured $\mathrm{T}_{2}$ bulk values were different: the $\mathrm{T}_{2}$ of the 
vacuole population protons before and after electroporation $765.2 \mathrm{~ms}$ and $433.8 \mathrm{~ms}$. A fast relaxing component $(2.58 \mathrm{~ms})$ is present. In the presence of a strongly inhomogeneous magnetic field, such as in the case of the Single-sided TD-NMR, the transverse relaxation time does not depend only on spin-spin interactions, but it is also controlled by molecular diffusion through the gradient, which results in a more rapid loss of coherence with the consequent shortening of the observed $\mathrm{T}_{2}$ values and the disappearance of very short $\mathrm{T}_{2}$ components. In potato cells, the lowest $T_{2}$ relaxation value can be assigned to water on the surface or inside starch granules [43]. Water in the cytoplasm, on cell walls, and in extracellular spaces shows overlapping $\mathrm{T}_{2}$ values between $30-40 \mathrm{~ms}$ and $250-300 \mathrm{~ms}$.

Electroporation causes a decrease of the $\mathrm{T}_{2}$ value associated with the vacuole water protons, as observed in surface $\mathrm{T}_{2}$ measurements. Starch water protons $\mathrm{T}_{2}$ values appear as a more disperse component, with a maximum $\mathrm{T}_{2}$ shifted to about $4.6 \mathrm{~ms}$.

After drying the potato in the oven, only the starch protons $\mathrm{T}_{2}$ component is still present (Fig. 8b), confirming the right attribution of the lowest $\mathrm{T}_{2}$ value to this cell compartment.

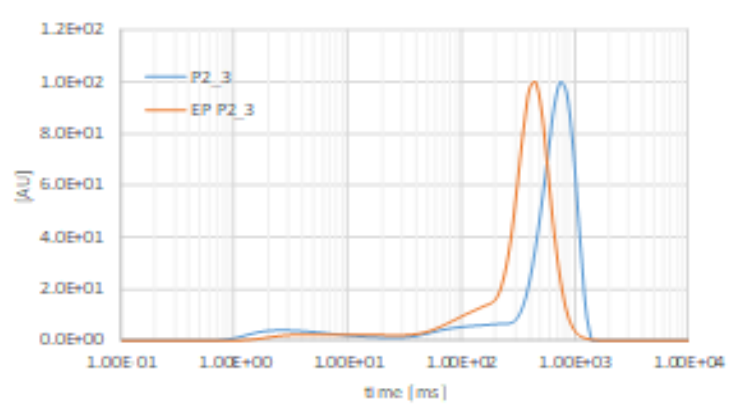

(a)

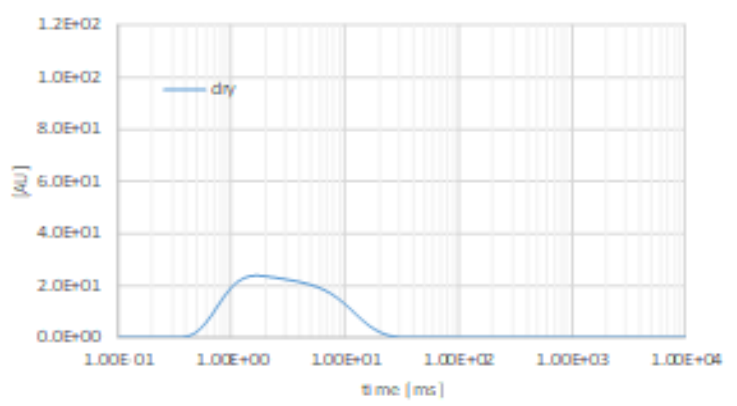

(b)

Figure 8. (a) T2 distribution of potato P2 before and after electroporation as measured with Minispec (b) T2 distribution in potato $\mathrm{P} 2$ after drying in the oven.

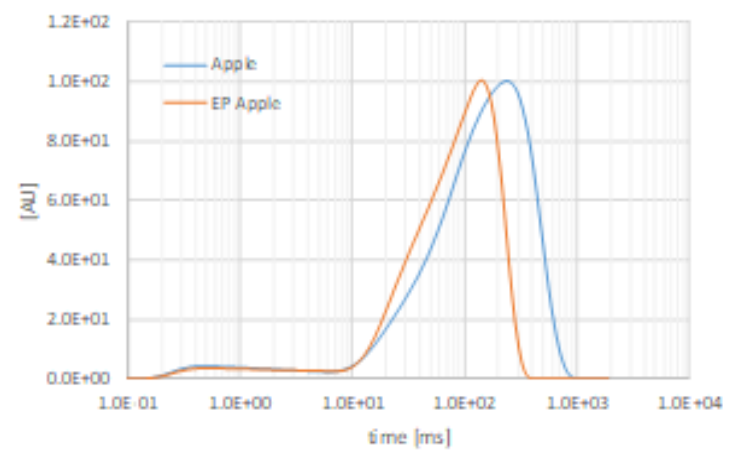

(a)

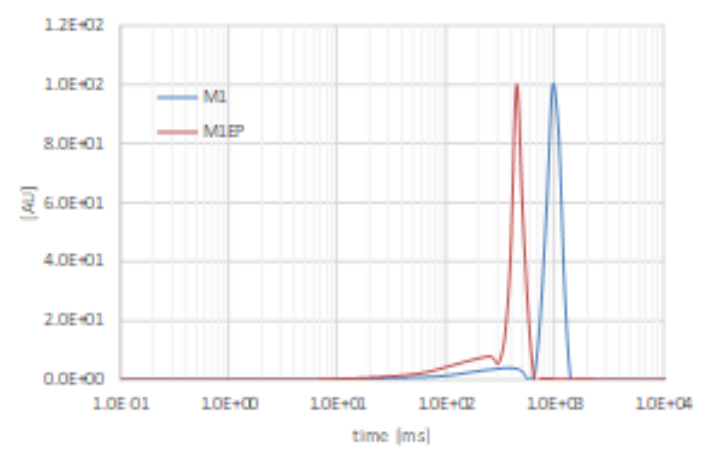

(b)

Figure 9. T2 distribution of the apple sample as measured with the Single-sided TD-NM0052 (a) and the Minispec (b).

NMR measurements on apple samples gave similar results to potato tissues. Fig 9a shows the $T_{2}$ distributions obtained before and after electroporation of apple with the Singlesided TD-NMR (side B) and Fig. 9b, with the Minispec. Using the Single-sided TD-NMR, the longest $\mathrm{T}_{2}$ value $(237.6 \mathrm{~ms})$ is higher than the one found in potatoes. This could be, probably because apples have bigger vacuoles than potatoes. Similar to what was found with potatoes, after electroporation, this peak is shifted to lower values (141.4 ms).

As expected, $\mathrm{T}_{2}$ values in a more homogeneous field are higher than in the inhomogeneous Single-sided TD-NMR. Water protons in vacuoles have a $\mathrm{T}_{2}$ relaxation time 
of $992.9 \mathrm{~ms}$, while after electroporation, the value decreases to $792.8 \mathrm{~ms}$. In comparison with potato proton populations, the absence of starch protons $\mathrm{T}_{2}$ component is evident. Correlations of $T_{2}$ values with vacuolar volumes and water loss were already reported in the literature $[39,42]$.

\section{Conclusions}

In this research, changes in potato tubers and apple tissues treated with electroporation were studied, evaluating the tissues' conductivity, taking microscope images, and analyzing their TD-NMR response.

The analysis using TD-NMR shows different behaviors in the distribution of the transverse relaxation time $\mathrm{T}_{2}$ values. The differences could be related to the modification in the structure of the cells that were damaged by voltage pulses and consequently lost some water originally stored inside their structure. This loss of internal water was also visible at a macroscopic level observing that the electroporated slices were wetter than the non-treated ones.

The $\mathrm{T}_{2}$ differences in treated and non-treated samples observed in this work may be explained with increased permeability of water pools across membranes due to the electroporation that affected the exchange rate among water protons from different compartments and leads to a loss of vacuolar water content.

The vacuole size reduction has been initially evidenced by decreasing the main $T_{2}$ value at the portable Single-sided TD-NMR and by the acquisition of images obtained at the electronic microscope.

A more accurate analysis of the transverse relaxation time distribution in potato and apple was performed using TD-NMR Minispec to confirm the relationship between the $\mathrm{T}_{2}$ values and the different compartments of the cells in which water is found. Moreover, the Minispec data of potato samples showed an interesting increase in the T2 value associated with the starch granules' protons upon electroporation. This can be explained by increased mobility of the starch in the cytoplasm after reducing the vacuolar volume.

Overall, comparing all the data obtained in the present work, TD-NMR's ability in monitoring subcellular water compartmentation proved to be valuable to confirm the cell changes after the electroporation process quickly, and there is a good correlation with previous literature.

\section{Funding}

This research received no external funding.

\section{Acknowledgments}

Roberta Bertani and MirtoMozzon wish to thank the TWINNING-2017 research project "Supramolecular transition-metal architectures based on non-covalent interactions for selective molecular sorption and non-linear optics: Rational design and preparation" of the Industrial Engineering Department (University of Padova) for the financial support. The authors are also grateful to Igea spa, Carpi (MO), Italy, for the pulse generator loan

\section{Conflicts of Interest}


The authors declare no conflict of interest.

\section{References}

1. Kotnik, T.; Kramar, P.; Pucihar, G.; Miklavčič, D.; Tarek, M. Cell membrane electroporation - Part 1: The phenomenon. IEEE Elect Insul Mag 2012, 28, 14-23, https://doi.org/10.1109/MEI.2012.6268438.

2. Zimmermann, U.; Pilwat, G.; Riemann, F. Dielectric breakdown of cell membranes. Biophy J 1974, 14, 881899, https://dx.doi.org/10.1016\%2FS0006-3495(74)85956-4.

3. Kotnik, T.; Rems, L.; Tarek, M.; Miklavčič, D. Membrane Electroporation and Electropermeabilization: Mechanisms and Models. Annu Rev Biophys 2019, 48, 63-91, https://doi.org/10.1146/annurev-biophys052118-115451.

4. Kiełbik, A.; Szlasa, W.; Saczko, J.; Kulbacka, J. Electroporation-Based Treatments in Urology. Cancers (Basel) 2020, 12, https://doi.org/10.3390/cancers12082208.

5. Sherba, J.J.; Hogquist, S.; Lin, H.; Shan, J.W.; Shreiber, D.I.; Zahn, J.D. The effects of electroporation buffer composition on cell viability and electro-transfection efficiency. Scientific Reports 2020, 10, 3053, 1-9. https://doi.org/10.1038/s41598-020-59790-x.

6. Cao, Y.; Ma, E.; Cestellos-Blanco, S.; Zhang, B.; Qiu, R.; Su, Y.; Doudna, J.A.; Yang, P. Nontoxic nanopore electroporation for effective intracellular delivery of biological macromolecules. Proceedings of the National Academy of Sciences 2019, 116, 7899-7904, https://doi.org/10.1073/pnas.1818553116.

7. Sachdev, S.; Feijoo Moreira, S.; Keehnen, Y.; Rems, L.; Kreutzer, M.T.; Boukany, P.E. DNA-membrane complex formation during electroporation is DNA size-dependent. Biochimica et Biophysica Acta (BBA) Biomembranes 2020, 1862, https://doi.org/10.1016/j.bbamem.2019.183089.

8. Tivig, I.; Savopol, T.; Kovacs, E.; Moisescu, M.G. An experimental system for real-time fluorescence recordings of cell membrane changes induced by electroporation. European biophysics journal 2020, 49, 105-111, https://doi.org/10.1007/s00249-019-01417-9.

9. Hoejholt, K.L.; Mužić, T.; Jensen, S.D.; Dalgaard, L.T.; Bilgin, M.; Nylandsted, J.; Heimburg, T.; Frandsen, S.K.; Gehl, J. Calcium electroporation and electrochemotherapy for cancer treatment: Importance of cell membrane composition investigated by lipidomics, calorimetry and in vitro efficacy. Scientific Reports 2019, 9, 1-12, https://doi.org/10.1038/s41598-019-41188-z.

10. Wu, Y.; Fu, A.; Yossifon, G. Active particles as mobile microelectrodes for selective bacteria electroporation and transport. Sci Adv 2020, 6, https://doi.org/10.1126/sciadv.aay4412.

11. Leontiadou, H.; Mark, A.E.; Marrink, S.J. Molecular dynamics simulations of hydrophilic pores in lipid bilayers. Biophys J 2004, 86, 2156-2164, https://doi.org/10.1016/S0006-3495(04)74275-7.

12. Rols, M.-P. Mechanism by Which Electroporation Mediates DNA Migration and Entry into Cells and Targeted Tissues. In: Electroporation Protocols: Preclinical and Clinical Gene Medicine. Li, S.; Ed. Humana Press: Totowa, NJ, 2008; pp. 19-33, https://doi.org/10.1007/978-1-59745-194-9_2.

13. Joshi, R.P.; Hu, Q.; Aly, R.; Schoenbach, K.H.; Hjalmarson, H.P. Self-consistent simulations of electroporation dynamics in biological cells subjected to ultrashort electrical pulses. Physical review. E, Statistical, non-linear, and soft matter physics 2001, 64, https://doi.org/10.1103/PhysRevE.64.011913.

14. Probst, U.; Fuhrmann, I.; Beyer, L.; Wiggermann, P. Electrochemotherapy as a New Modality in Interventional Oncology: A Review. Technology in cancer research \& treatment 2018, 17, https://doi.org/10.1177/1533033818785329.

15. Marty, M.; Sersa, G.; Garbay, J.R.; Gehl, J.; Collins, C.G.; Snoj, M.; Billard, V.; Geertsen, P.F.; Larkin, J.O.; Miklavcic, D.; Pavlovic, I.; Paulin-Kosir, S.M.; Cemazar, M.; Morsli, N.; Soden, D.M.; Rudolf, Z.; Robert, C.; O’Sullivan, G.C.; Mir, L.M. Electrochemotherapy - An easy, highly effective and safe treatment of cutaneous and subcutaneous metastases: Results of ESOPE (European Standard Operating Procedures of Electrochemotherapy) study. European Journal of Cancer Supplements 2006, 4, 3-13, https://doi.org/10.1016/j.ejcsup.2006.08.002.

16. Ivorra, A.; Al-Sakere, B.; Rubinsky, B.; Mir, L.M. In vivo electrical conductivity measurements during and after tumor electroporation: conductivity changes reflect the treatment outcome. Physics in medicine and biology 2009, 54, 5949-5963, https://doi.org/10.1088/0031-9155/54/19/019.

17. Miklavčič, D. Handbook of Electroporation. 2016.

18. Rebersek, M.; Miklavcic, D.; Bertacchini, C.; Sack, M. Cell membrane electroporation-Part 3: the equipment. IEEE Electrical Insulation Magazine $\mathbf{2 0 1 4}, \quad 30, \quad 8-18$, https://doi.org/10.1109/MEI.2014.6804737.

19. Sampedro, F.; Rodrigo, D. Pulsed Electric Fields (PEF) Processing of Milk and Dairy Products. In: Emerging Dairy Processing Technologies. Opp Dai Ind 2015; pp. 115-148, https://doi.org/10.1002/9781118560471.ch5.

20. Zhao, W.; Tang, Y.; Lu, L.; Chen, X.; Li, C. Review: Pulsed Electric Fields Processing of Protein-Based Foods. Food and Bioprocess Technology 2014, 7, 114-125, https://doi.org/10.1007/s11947-012-1040-1.

21. Mir, L.M.; Glass, L.F.; Sersa, G.; Teissié, J.; Domenge, C.; Miklavcic, D.; Jaroszeski, M.J.; Orlowski, S.; Reintgen, D.S.; Rudolf, Z.; Belehradek, M.; Gilbert, R.; Rols, M.P.; Belehradek, J., Jr.; Bachaud, J.M.; DeConti, R.; Stabuc, B.; Cemazar, M.; Coninx, P.; Heller, R. Effective treatment of cutaneous and 
subcutaneous malignant tumours by electrochemotherapy. British journal of cancer 1998, 77, 2336-2342, https://doi.org/10.1038/bjc.1998.388.

22. Saulis, G. Electroporation of Cell Membranes: The Fundamental Effects of Pulsed Electric Fields in Food Processing. Food Engineering Reviews 2010, 2, 52-73, https://doi.org/10.1007/s12393-010-9023-3.

23. Donsì, G.; Ferrari, G.; Pataro, G. Inactivation kinetics of Saccharomyces cerevisiae by pulsed electric fields in a batch treatment chamber: The effect of electric field unevenness and initial cell concentration. Journal of Food Engineering 2007, 78, 784-792, https://doi.org/10.1016/j.jfoodeng.2005.11.027.

24. Toepfl, S.; Heinz, V.; Knorr, D. High intensity pulsed electric fields applied for food preservation. Chemical Engineering and Processing: Process Intensification 2007, 46, 537-546, https://doi.org/10.1016/j.cep.2006.07.011.

25. Eveke, D.J.G.; Brunkhorst, C. Inactivation of in Apple Juice by Radio Frequency Electric Fields. Journal of Food Science 2006, 69, FEP134-FEP0138, https://doi.org/10.1111/j.1365-2621.2004.tb13366.x.

26. Zhang, Q.; Monsalve-GonzÁLez, A.; Qin, B.-L.; Barbosa-CÁNovas, G.V.; Swanson, B.G. Inactivation Of Saccharomyces Cerevisiae In Apple Juice By Square-Wave And Exponential-Decay Pulsed Electric Fields. Journal of Food Process Engineering 1994, 17, 469-478, https://doi.org/10.1111/j.17454530.1994.tb00350.x.

27. Bertram, H.C.; Purslow, P.P.; Andersen, H.J. Relationship between Meat Structure, Water Mobility, and Distribution: A Low-Field Nuclear Magnetic Resonance Study. J Agric Food Chem 2002, 50, 824-829, https://doi.org/10.1021/jf010738f.

28. Proietti, N.; Adiletta, G.; Russo, P. Evolution of physicochemical properties of pear during drying by conventional techniques, portable-NMR, and modelling. Journal of Food Engineering 2018, 230, 82-98, https://doi.org/10.1016/j.jfoodeng.2018.02.028.

29. Menegazzo, I.; Mammi, S.; Sgarbossa, P.; Bartolozzi, A.; Mozzon, M.; Bertani, R.; Forzan, M.; Sundararajan, R.; Sieni, E. Time Domain Nuclear Magnetic Resonance (TD-NMR) to evaluate the effect of potato cell membrane electroporation. Innovative Food Science \& Emerging Technologies 2020, 65, https://doi.org/10.1016/j.ifset.2020.102456.

30. Besghini, D.; Mauri, M.; Simonutti, R. Time Domain NMR in Polymer Science: From the Laboratory to the Industry. Applied Sciences 2019, 9, https://doi.org/10.3390/app9091801.

31. Nikolskaya, E.; Hiltunen, Y. Time-Domain NMR in Characterization of Liquid Fuels: A Mini-Review. Energy \& Fuels 2020, 34, 7929-7934, https://doi.org/10.1021/acs.energyfuels.0c01464.

32. Tang, Y.; McCowan, D.; Song, Y.-Q. A miniaturized spectrometer for NMR relaxometry under extreme conditions. Scientific Reports 2019, 9, 1-9, https://doi.org/10.1038/s41598-019-47634-2.

33. Relaxation NMR. Available online: https://www.imaios.com/en/e-Courses/e-MRI/NMR/Relaxation-nmr (accessed on 7 Jan 2021).

34. Parenti, O.; Guerrini, L.; Zanoni, B.; Marchini, M.; Tuccio, M.G.; Carini, E. Use of the 1H NMR technique to describe the kneading step of wholewheat dough: The effect of kneading time and total water content. Food Chemistry 2021, 338, https://doi.org/10.1016/j.foodchem.2020.128120.

35. Crook, A.A.; Powers, R. Quantitative NMR-Based Biomedical Metabolomics: Current Status and Applications. Molecules 2020, 25, https://doi.org/10.3390/molecules25215128.

36. Smits, J.; Damron, J.T.; Kehayias, P.; McDowell, A.F.; Mosavian, N.; Fescenko, I.; Ristoff, N.; Laraoui, A.; Jarmola, A.; Acosta, V.M. Two-dimensional nuclear magnetic resonance spectroscopy with a microfluidic diamond quantum sensor. Science Advances 2019, 5, https://doi.org/10.1126/sciadv.aaw7895.

37. Bartusik-Aebisher, D.; Aebisher, D.; Czmil, M.; Mazur, D. Evaluation of MR relaxation times following trastuzumab treatment of breast cancer cells in a 3D bioreactor. Acta Poloniae Pharmaceutica - Drug Research 2020, 77, 35-41, https://doi.org/10.32383/appdr/115519.

38. Sorte, E.G.; Rimsza, J.M.; Alam, T.M. Computational and Experimental 1H-NMR Study of Hydrated MgBased Minerals. Molecules 2020, 25, https://doi.org/10.3390/molecules25040933.

39. Lin, X.; Zhan, H.; Li, H.; Huang, Y.; Chen, Z. NMR Relaxation Measurements on Complex Samples Based on Real-Time Pure Shift Techniques. Molecules 2020, 25, https://doi.org/10.3390/molecules25030473.

40. Duynhoven, J.; Voda, M.; Witek, M.; As, H. Time-Domain NMR Applied to Food Products. Annual Reports on NMR Spectroscopy 2010, 69, 145-197, https://doi.org/10.1016/S0066-4103(10)69003-5.

41. Snaar, J.E.M.; Van As, H. Probing water compartments and membrane permeability in plant cells by $1 \mathrm{H}$ NMR relaxation measurements. Biophys $J$ 1992, 63, 1654-1658, https://doi.org/10.1016/S00063495(92)81741-1.

42. Hills, B.P.; Remigereau, B. NMR studies of changes in subcellular water compartmentation in parenchyma apple tissue during drying and freezing. International Journal of Food Science \& Technology 1997, 32, 5161, https://doi.org/10.1046/j.1365-2621.1997.00381.x.

43. Hansen, C.L.; Thybo, A.K.; Bertram, H.C.; Viereck, N.; van den Berg, F.; Engelsen, S.B. Determination of Dry Matter Content in Potato Tubers by Low-Field Nuclear Magnetic Resonance (LF-NMR). J Agric Food Chem 2010, 58, 10300-10304, https://doi.org/10.1021/jf101319q.

44. Capitani, D.; Sobolev, A.P.; Delfini, M.; Vista, S.; Antiochia, R.; Proietti, N.; Bubici, S.; Ferrante, G.; Carradori, S.; De Salvador, F.R.; Mannina, L. NMR methodologies in the analysis of blueberries. Electrophoresis 2014, 35, 1615-1626, https://doi.org/10.1002/elps.201300629. 
45. Capitani, D.; Mannina, L.; Proietti, N.; Sobolev, A.P.; Tomassini, A.; Miccheli, A.; Di Cocco, M.E.; Capuani, G.; De Salvador, R.; Delfini, M. Monitoring of metabolic profiling and water status of Hayward kiwifruits by nuclear magnetic resonance. Talanta 2010, 82, 1826-1838, https://doi.org/10.1016/j.talanta.2010.07.080.

46. Raffo, A.; Gianferri, R.; Barbieri, R.; Brosio, E. Ripening of banana fruit monitored by water relaxation and diffusion 1H-NMR measurements. Food Chemistry 2005, 89, 149-158, https://doi.org/10.1016/j.foodchem.2004.02.024.

47. Imakumbili, M.L.E.; Semu, E.; Semoka, J.M.R.; Abass, A.; Mkamilo, G. Plant tissue analysis as a tool for predicting fertiliser needs for low cyanogenic glucoside levels in cassava roots: An assessment of its possible use. PLoS One 2020, 15, https://doi.org/10.1371/journal.pone.0228641.

48. Blümich, B.; Perlo, J.; Casanova, F. Mobile single-sided NMR. Prog Nuc Mag Reson Spect 2008, 52, 197269, https://doi.org/10.1016/j.pnmrs.2007.10.002.

49. Capitani, D.; Sobolev, A.P.; Di Tullio, V.; Mannina, L.; Proietti, N. Portable NMR in food analysis. Chemical and Biological Technologies in Agriculture 2017, 4, https://doi.org/10.1186/s40538-017-0100-1.

50. Bernardis, A.; Bullo, M.; Campana, L.G.; Di Barba, P.; Dughiero, F.; Forzan, M.; Mognaschi, M.E.; Sgarbossa, P.; Sieni, E. Electric field computation and measurements in the electroporation of inhomogeneous samples. Open Physics 2017, 15, 790-796, https://doi.org/10.1515/phys-2017-0092.

51. Bertacchini, C.; Margotti, P.M.; Bergamini, E.; Lodi, A.; Ronchetti, M.; Cadossi, R. Design of an irreversible electroporation system for clinical use. Technology in cancer research \& treatment 2007, 6, 313-320, https://doi.org/10.1177/153303460700600408.

52. Breton, M.; Buret, F.; Krähenbühl, L.; Leguèbe, M.; Mir, L.; Perrussel, R.; Poignard, C.; Scorretti, R.; Voyer, D. Non-Linear Steady-State Electrical Current Modeling for the Electropermeabilization of Biological Tissue. IEEE Transactions on Magnetics 2015, 51, 1-4, https://doi.org/10.1109/TMAG.2014.2351836.

53. Campana, L.G.; Cesari, M.; Dughiero, F.; Forzan, M.; Rastrelli, M.; Rossi, C.R.; Sieni, E.; Tosi, A.L. Electrical resistance of human soft tissue sarcomas: an ex vivo study on surgical specimens. Med Biol Eng Comput 2016, 54, 773-787, https://doi.org/10.1007/s11517-015-1368-6.

54. Sarnago, H.; Sieni, E.; Lopez-Alonso, B.; Carrctcro, C.; Burdio, J.M.; Ó, L. An Inter-Disciplinary Approach to Teaching Biomedical Electronics with an Electroporation-Applied Example. In: Proceedings of 2018 IEEE 27th International Symposium on Industrial Electronics (ISIE). 13-15 June 2018; pp. 901-905.

55. Carr, H.Y.; Purcell, E.M. Effects of Diffusion on Free Precession in Nuclear Magnetic Resonance Experiments. Physical Review 1954, 94, 630-638, https://doi.org/10.1103/PhysRev.94.630.

56. Meiboom, S.; Gill, D. Modified Spin-Echo Method for Measuring Nuclear Relaxation Times. Review of Scientific Instruments 1958, 29, 688-691, https://doi.org/10.1063/1.1716296.

57. Borgia, G.C.; Brown, R.J.S.; Fantazzini, P. Uniform-Penalty Inversion of Multiexponential Decay Data: II. Data Spacing, T2 Data, Systematic Data Errors, and Diagnostics. Journal of Magnetic Resonance 2000, 147, 273-285, https://doi.org/10.1006/jmre.2000.2197.

58. Borgia, G.C.; Brown, R.J.S.; Fantazzini, P. Uniform-Penalty Inversion of Multiexponential Decay Data. Journal of Magnetic Resonance 1998, 132, 65-77, https://doi.org/10.1006/jmre.1998.1387.

59. Venditti, G.; Schievano, E.; Navarini, L.; Mammi, S. Water Mobility and Distribution in Green Coffee Probed by Time-Domain Nuclear Magnetic Resonance. Food Biophysics 2011, 6, 321-326, https://doi.org/10.1007/s11483-010-9194-8.

60. Ivorra, A.; Mir, L.M.; Rubinsky, B. Electric Field Redistribution due to Conductivity Changes during Tissue Electroporation: Experiments with a Simple Vegetal Model. In: Proceedings of World Congress on Medical Physics and Biomedical Engineering. September 7 - 12, 2009, Munich, Germany, Berlin, Heidelberg, 2010; pp. 59-62.

61. Hjouj, M.; Rubinsky, B. Magnetic resonance imaging characteristics of nonthermal irreversible electroporation in vegetable tissue. The Journal of membrane biology 2010, 236, 137-146, https://doi.org/10.1007/s00232-010-9281-2.

62. Knorr, D.; Angersbach, A. Impact of high-intensity electric field pulses on plant membrane permeabilization. Trends in Food Science \& Technology 1998, 9, 185-191, https://doi.org/10.1016/S0924-2244(98)00040-5.

63. Galindo, F.G.; Vernier, P.T.; Dejmek, P.; Vicente, A.; Gundersen, M.A. Pulsed electric field reduces the permeability of potato cell wall. Bioelectromagnetics 2008, 29, 296-301, https://doi.org/10.1002/bem.20394.

64. Angersbach, A.; Heinz, V.; Knorr, D. Electrophysiological model of intact and processed plant tissues: cell disintegration criteria. Biotechnol Prog 1999, 15, 753-762, https://doi.org/10.1021/bp990079f.

65. Angersbach, A.; Heinz, V.; Knorr, D. Effects of pulsed electric fields on cell membranes in real food systems. Innovative Food Science \& Emerging Technologies 2000, 1, 135-149, https://doi.org/10.1016/S14668564(00)00010-2.

66. Gehl, J. Electroporation: theory and methods, perspectives for drug delivery, gene therapy and research. Acta physiologica Scandinavica 2003, 177, 437-447, https://doi.org/10.1046/j.1365-201X.2003.01093.x. 